\title{
Investigating trajectories of social recovery in individuals with first-episode psychosis: a latent class growth analysis
}

Jo Hodgekins, Max Birchwood, Rose Christopher, Max Marshall, Sian Coker, Linda Everard, Helen Lester, ${ }^{*}$ Peter Jones, Tim Amos, Swaran Singh, Vimal Sharma, Nick Freemantle and David Fowler

\section{Background}

Social disability is a hallmark of severe mental illness yet individual differences and factors predicting outcome are largely unknown.

\section{Aim}

To explore trajectories and predictors of social recovery following a first episode of psychosis (FEP).

\section{Method}

A sample of 764 individuals with FEP were assessed on entry into early intervention in psychosis (EIP) services and followed up over 12 months. Social recovery profiles were examined using latent class growth analysis.

\section{Results}

Three types of social recovery profile were identified: Low Stable (66\%), Moderate-Increasing (27\%), and High-Decreasing (7\%). Poor social recovery was predicted by male gender, ethnic minority status, younger age at onset of psychosis, increased negative symptoms, and poor premorbid adjustment.

\section{Conclusions}

Social disability is prevalent in FEP, although distinct recovery profiles are evident. Where social disability is present on entry into EIP services it can remain stable, highlighting a need for targeted intervention.

\section{Declaration of interest}

None.

\section{Copyright and usage}

(c) The Royal College of Psychiatrists 2015. This is an open access article distributed under the terms of the creative commons Attribution (CC BY) licence.
Social disability refers to difficulties with social and occupational functioning (i.e. difficulties engaging in meaningful activities and relationships), and has been described as a hallmark of severe mental illness. ${ }^{1}$ There is a large social cost attached to such disability, with a substantial proportion of the estimated cost of psychosis attributed to unemployment and lost productivity

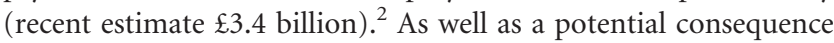
of psychosis, it has been suggested that social disability may precede illness onset, with functional impairment also evident in the prodromal phase. ${ }^{3}$

Many aspects of social functioning are affected by psychosis, including employment, relationships and recreational activities. Social and functional outcomes in psychosis are frequently reported to be poor, with follow-up studies suggesting less than $50 \%$ of people with a non-affective psychosis achieve a social recovery, with even fewer returning to competitive employment in the long-term. ${ }^{4}$

Evidence suggests that early intervention in psychosis (EIP) may have a positive impact on functional outcome, ${ }^{5}$ but few studies include this area of recovery as a primary outcome and further research is necessary. In addition, problems with measuring social and functional recovery lead to variation in the rates of social disability and recovery reported within the literature. ${ }^{6}$ Measures of social functioning are often confounded with psychotic symptoms, and many were designed for use with individuals with chronic schizophrenia rather than those in the early stages of psychosis. Further research is needed using more valid and accurate assessments of functioning to examine rates and patterns of social recovery over time.

From a research perspective, recovery from first-episode psychosis (FEP) currently tends to be treated as a homogeneous construct, with studies investigating predictors of outcome using

*Deceased group means on measures of functioning, or by comparing FEP samples with non-clinical comparison groups. ${ }^{7}$ Rather than all individuals responding to psychosis in the same way, it is arguably more likely that cohorts of individuals with FEP are heterogeneous, consisting of subgroups with different baseline levels of social disability and different recovery pathways. Identifying different patterns of recovery will be important in developing and implementing targeted recovery-focused interventions. This approach also has face validity in relation to observations of heterogeneity from clinical practice. In the current study, social recovery over a 12-month period following FEP is examined using latent class growth analysis (LCGA) to examine subgroups of individuals with different recovery trajectories. Predictors of these different trajectories are then examined.

\section{Method}

Longitudinal data from the National EDEN study were used for the analyses described in this paper. National EDEN is a national evaluation of EIP services across the UK (services in: Birmingham, Norwich, Cambridge, Cornwall, Bristol and Lancashire), funded by the Department of Health. ${ }^{8}$ The aim of National EDEN was to evaluate the implementation, effectiveness and cost-effectiveness of the first 12 months of care provided by EIP services in the UK. EIP services provide dedicated care to young people aged 14-35 who are experiencing a first episode of psychosis. Further details of care provided by these teams is described in detail in the Policy Implementation Guide. ${ }^{9}$ Consecutive patients accepted into each EIP service from August 2005 to April 2009 were approached and invited to take part in the study. Participants were assessed up to 3 months post acceptance into EIP (baseline), and 6 and 12 months later. A total of 1027 individuals consented to 
take part, with $80 \%$ followed up at 6 months and $77 \%$ followed up at 12 months.

\section{Participants}

The sample included in this study are 764 participants (74\%) from the National EDEN study who completed the Time Use Survey (TUS) at baseline and at least one other time point (6 months and/or 12 months). Participants who completed the TUS did not differ from participants who did not in terms of age at onset of psychosis, diagnosis, duration of untreated psychosis (DUP), gender, ethnicity and work status. Demographic information about the sample is provided in Table 1.

\section{Measures}

Time use survey (TUS) ${ }^{10,11}$

Social recovery was assessed using the TUS at baseline, 6 months and 12 months to measure weekly hours engaged in structured activity. The TUS administered in the study was a shortened version of the individual questionnaire originally used by the Office for National Statistics, ${ }^{10}$ in a national survey to examine how members of the population of the UK spend their time.

Categories of activity included in the TUS are: work, education, voluntary work, housework and childcare, leisure, and sports. Lists of activities are provided for each category (e.g. leisure activities include going to the cinema, pub and eating out). Participants are asked how many times they had engaged in each activity over the past month and for how long on each occasion. A weekly average is then calculated for hours spent in structured activity over the past month (paid/voluntary work, education, childcare and chores, and structured social activities). In the current study, the TUS was administered by trained interviewers, taking approximately $20 \mathrm{~min}$ to complete (interrater reliability, intraclass correlation $=0.99$ ).

On average, a non-clinical sample aged between 16 and 36 years spends $63.5 \mathrm{~h}$ per week in structured activity (data from the Office for National Statistics). ${ }^{10}$ The TUS has been successfully used with a sample of individuals with psychosis enabling clinical cut-off scores to be established..$^{11}$ A cut-off score of $45 \mathrm{~h}$ per week is indicative of good social functioning (i.e. within the non-clinical range). Individuals scoring between 30 and $45 \mathrm{~h}$ per week can be defined as at-risk of social disability. Individuals scoring below $30 \mathrm{~h}$ per week can be defined as experiencing social disability; and individuals scoring below $15 \mathrm{~h}$ per week can be defined as experiencing severe social disability.

\begin{tabular}{|c|c|}
\hline \multicolumn{2}{|l|}{ Characteristic } \\
\hline Age at onset, years: mean (s.d.) & $21.29(5.03)$ \\
\hline Duration of untreated psychosis $>4$ months, $n(\%)$ & $320(41.9)$ \\
\hline \multicolumn{2}{|l|}{ Diagnosis, $n(\%)$} \\
\hline Psychosis & $595(77.9)$ \\
\hline Schizophrenia & $70(9.2)$ \\
\hline Bipolar/schizoaffective disorder & $49(6.4)$ \\
\hline \multicolumn{2}{|l|}{ Gender, $n$ (\%) } \\
\hline Male & $532(69.6)$ \\
\hline Female & $232(30.4)$ \\
\hline \multicolumn{2}{|l|}{ Ethnicity, $n(\%)$} \\
\hline White & $556(72.8)$ \\
\hline Asian & $116(15.2)$ \\
\hline Black Caribbean & $53(6.9)$ \\
\hline Mixed ethnicity & $39(5.1)$ \\
\hline Not in education, employment or training, $n$ (\%) & $437(57.2)$ \\
\hline
\end{tabular}

\section{Candidate explanatory variables}

Potential explanatory variables were assessed at baseline. DUP was assessed retrospectively using the method described by Larsen et $a l^{12}$ using notes and participant reports to ascertain the length of time between the onset of psychotic symptoms and the start of criterion treatment. A dichotomous DUP variable was then constructed representing short $(<4$ months) and long ( $>4$ months) DUP based on suggestions in the current literature that there is likely to be a 'critical period' of DUP. ${ }^{13}$ The Premorbid Adjustment Scale (PAS) $)^{14}$ was used to assess participants' self-reported functioning prior to the onset of their psychotic episode in childhood (up to age 11), early adolescence (12-15 years), and late adolescence (16-18 years). The Positive and Negative Syndrome Scale (PANSS) ${ }^{15}$ was used to assess the frequency and severity of psychotic symptomatology (positive, negative, and general) and the Calgary Depression Scale (CDS) ${ }^{16}$ was used to assess symptoms of depression.

\section{Analysis plan}

Identifying trajectories of social recovery

Latent class growth analysis (LCGA) is a technique developed by Nagin $^{17}$ for identifying distinct homogeneous subpopulations with similar trajectories of growth over time (known as latent classes) within longitudinal data collected from a larger heterogeneous population. ${ }^{18}$ LCGA is a type of growth mixture modelling where the variance and covariance estimates for growth factors within each class are fixed to zero, assuming homogeneity within classes. ${ }^{19}$ The analyses were conducted using Mplus version $4 .{ }^{20}$ As data were only available for three time points, only linear growth curves could be fitted. Models with varying numbers of latent classes were fitted to the data, increasing the number of classes until the model which best fitted the data was identified. The best-fitting model was chosen according to the following fit indices: Bayesian Information Criterion (BIC), Lo-Mendell-Rubin Likelihood Ratio Test (LMR-LRT), and Bootstrapped Likelihood Ratio Test (BLRT). Lower BIC values suggest more parsimonious model fit, whereas a significant LMR-LRT or BLRT value suggests that a $\mathrm{K}$ class model fits the data better than a K-1 model, i.e. an additional class improves model fit. Interpretability of the successive models were also considered alongside fit indices.

\section{Examining predictors of social recovery.}

The estimated recovery trajectory classes were saved and imported into SPSS version $16^{21}$ for further exploratory analyses. Classes of individuals with different social recovery trajectories were compared on baseline predictor variables using one-way ANOVAs and chi-squared tests. Post hoc tests were conducted to interpret significant main effects. Multinomial regression was used to examine predictors of recovery trajectory, with social recovery trajectory as the response variable (reference category = Low Stable trajectory) and candidate explanatory variables including: gender; ethnicity; DUP; age at onset of psychosis; positive, negative, and general psychotic symptoms; depression; and premorbid adjustment. Multinomial regression was used as the recovery trajectories were treated as nominal, rather than ranked ordinal, categorical variables. A Bonferroni corrected $P$-value of $P<0.004$ $(0.05 / 14)$ was used to establish statistical significance when conducting multiple comparisons, to correct for family-wise error.

\section{Results}

\section{Descriptive statistics}

Descriptive statistics for TUS scores at each of the three time points and predictor variables at baseline are shown in Table 2. 


\begin{tabular}{|c|c|c|c|c|}
\hline & $n$ & Minimum-maximum & Median & Mean (s.d.) \\
\hline \multicolumn{5}{|c|}{ Structured activity, hours per week } \\
\hline Baseline & 764 & $0-140$ & 15.25 & $25.07(26.23)$ \\
\hline 6 months & 673 & $0-140$ & 24.00 & $30.82(25.28)$ \\
\hline 12 months & 623 & $0-136$ & 26.50 & $32.49(26.97)$ \\
\hline PANSS positive symptoms & 727 & $7-33$ & 15.00 & $15.09(5.87)$ \\
\hline PANSS negative symptoms & 718 & $7-43$ & 14.00 & $15.14(6.56)$ \\
\hline PANSS general symptoms & 722 & $16-79$ & 32.00 & $32.94(9.96)$ \\
\hline Calgary Depression Scale & 736 & $0-26$ & 5.00 & $6.37(5.36)$ \\
\hline \multicolumn{5}{|l|}{ Premorbid adjustment } \\
\hline Childhood & 715 & $0-0.88$ & 0.21 & $0.23(0.18)$ \\
\hline Early adolescence & 691 & $0-0.77$ & 0.27 & $0.29(0.17)$ \\
\hline Late adolescence & 586 & $0-0.93$ & 0.30 & $0.31(0.19)$ \\
\hline
\end{tabular}

The proportion of individuals scoring on each of the specified cut-offs on the TUS at each time point is shown in the online data supplement (Table DS1).

\section{Latent classes of recovery}

Growth models with varying numbers of latent classes were fitted to the data, increasing the number of classes until the model which best fitted the data was identified. Table 3 shows the model fit for all LCGA models assessed. Overall, a three-class model described the data well and was a significantly better fit than a model with two classes, according to both the LMR-LRT and the BLRT statistics. Although the BIC value reduced further for models with four and more classes, and these models were deemed a better fit according to the BLRT, a three-class model was chosen for reasons of parsimony and interpretability. Models with four and more classes included classes consisting of less than $5 \%$ of the sample and did not add any further interpretive value. This was also supported by a non-significant LMR-LRT value for models with four and more classes.

Average class probabilities for the three-class model were high (0.84-0.94), indicating participants were correctly assigned to their respective latent classes. Convergence checks were conducted on the three-class model to ensure that it was not a local solution. ${ }^{18}$ Model estimates were replicated, suggesting a global solution and increasing the stability of the findings. Thus, a three-class model was chosen as the best fitting model and is illustrated in Fig. 1.

\section{Class 1 - low stable time use}

The first class contained the largest number of participants ( $n=507,66.3 \%$ of the sample) and was characterised by a trajectory with a low intercept (unstandardised mean intercept $=14.00)$ and a shallow but significant positive slope (unstandardised mean slope $=3.18, P=0.001$ ). This class was labelled the Low Stable group, reflecting individuals with high levels of social disability at baseline which remained relatively stable and below the non-clinical range over the study period.

\section{Class 2 - moderate-increasing time use}

The second class ( $n=204,26.7 \%$ of the sample) was characterised by a trajectory with a moderate intercept (unstandardised mean intercept $=36.64$ ) and a significant positive slope (unstandardised mean slope $=10.29, \quad P<0.001)$. This class was labelled the Moderate-Increasing group, reflecting individuals with a moderate level of social disability at baseline which improved over the study period into the non-clinical range.

\section{Class 3 - high-decreasing time use}

The third class ( $n=53,6.9 \%$ of the sample) was characterised by a trajectory with a high intercept (unstandardised mean intercept $=90.53$ ) and a significant negative slope (unstandardised mean slope $=-12.78, P=0.008)$. This class was labelled the High-Decreasing group, reflecting individuals who were not socially disabled at baseline (scoring above the non-clinical range) and, despite a large decrease, maintained their level of functioning within the non-clinical range throughout the 12-month study period. Nonetheless, there was a significant deterioration in time spent in structured activity in this group which could become problematic if it did not stabilise following the 12-month period.

\section{Between-group differences}

The three classes (Low Stable, Moderate-Increasing and HighDecreasing) were compared on demographic characteristics and baseline predictor variables. Descriptive statistics are shown in

Table 3 Criteria for deciding the number of classes within the repeated measures of time use

\begin{tabular}{|c|c|c|c|c|c|c|}
\hline Number of classes & $\begin{array}{c}\text { Number of free } \\
\text { parameters }\end{array}$ & $\mathrm{BIC}$ & LMR-LRT statistic & LMR-LRT $P$ & BLRT statistic & BLRT $P$ \\
\hline 1 & 5 & 18851.91 & - & - & - & - \\
\hline 2 & 8 & 18809.33 & 508.57 & $<0.001$ & 534.11 & $<0.001$ \\
\hline 3 & 11 & 18652.52 & 168.28 & 0.05 & 176.73 & $<0.001$ \\
\hline 4 & 14 & 18582.12 & 86.00 & 0.32 & 90.32 & $<0.001$ \\
\hline 5 & 17 & 18506.09 & 91.36 & 0.10 & 95.94 & $<0.001$ \\
\hline 6 & 20 & 18452.27 & 70.76 & 0.27 & 74.32 & $<0.001$ \\
\hline
\end{tabular}




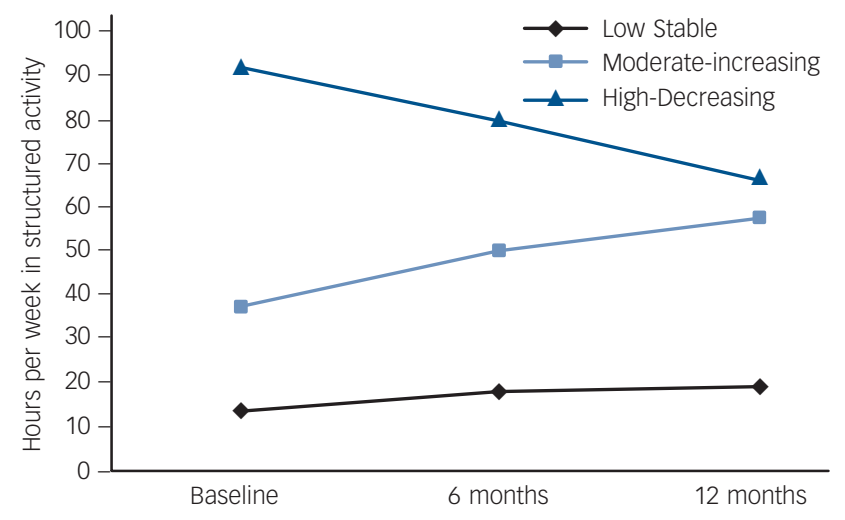

Fig. 1 Latent class growth analysis model with three social recovery trajectories.

Table 4. Group differences were found in age at onset of psychosis, gender, ethnicity, psychotic symptoms and premorbid adjustment. The Low Stable group had a younger age at onset of their psychosis, higher baseline levels of psychotic symptoms (positive, negative and general), and poorer premorbid adjustment in adolescence. There were also a higher proportion of males and individuals from ethnic minority groups in the low stable group. There were no group differences in DUP, baseline depression scores or premorbid adjustment in childhood up to 11 years. After applying a Bonferroni correction for multiple comparisons $(P<0.004)$, all between-group differences remained significant.

\section{Predictors of recovery}

Baseline predictor variables were entered into a multinomial regression model with recovery latent class as the dependent variable, using the Low Stable recovery trajectory as the reference category. The results of the regression model are shown in Table 5.

Compared with individuals with a low stable trajectory, individuals with a moderate-increasing trajectory were more likely to be female, less likely to have ethnic minority status, have lower levels of positive symptoms at baseline, and better premorbid adjustment (lower scores on the PAS) in late adolescence.

Compared with individuals with a low stable trajectory, individuals with a high-decreasing trajectory were also more likely to be female and to have better premorbid adjustment in late adolescence. In addition, they were more likely to have lower baseline negative symptoms and an older age at onset of psychosis. Interestingly, individuals with a high-decreasing trajectory were also more likely to have poorer premorbid adjustment (higher scores on the PAS) in childhood, compared with those with a low stable trajectory.

The results suggest that being male and having an ethnic minority status may be associated with a poorer social recovery trajectory. Moreover, high baseline levels of negative symptoms, poor premorbid adjustment in adolescence, and a younger age at onset of psychosis may also increase the likelihood of a poor functional outcome.

\section{Discussion}

\section{Summary of results}

This study utilised LCGA to examine trajectories of social functioning in a large longitudinal dataset. The results suggest that social recovery from FEP is heterogeneous. A large proportion of individuals displayed a high level of social disability which did not improve over the first 12 months of service provision (66\%). However, there was also a minority who did not display social disability at baseline or follow-up, scoring above the non-clinical cut-off on the TUS, but who nevertheless experienced a significant reduction in time use over the 12-month period (7\%). A further group experienced moderate levels of social disability when presenting with their first episode of psychosis but demonstrated an improvement in functioning over the 12-month period (27\%). Factors predicting poor social recovery over time included male gender, poor adolescent premorbid adjustment, high baseline levels of negative symptoms, ethnic minority status, and a younger age at onset of psychosis.

\section{Interpretation of findings}

\section{Rates of recovery}

Participants in the Moderate-Increasing group (27\%) had a good outcome, with their functioning improving over time and reaching the non-clinical range at the end of the 12-month period. It could be argued that participants in the High-Decreasing group

\begin{tabular}{|c|c|c|c|c|}
\hline & $\begin{array}{l}\text { Low Stable } \\
\qquad(n=507)\end{array}$ & $\begin{array}{l}\text { Moderate-Increasing } \\
\qquad(n=204)\end{array}$ & $\begin{array}{l}\text { High-Decreasing } \\
\qquad(n=53)\end{array}$ & Statistic \\
\hline Age at onset of psychosis, years: mean (s.d.) & $20.75(4.67)^{\star}$ & $21.88(5.32) * *$ & $24.30(5.97)^{\star \star *}$ & $F(2,728)=13.58, P<0.001$ \\
\hline Male, $n(\%)$ & $378(75)^{*}$ & $128(63)^{* *}$ & $26(49)^{\star *}$ & $\chi^{2}(2)=21.00, P=<0.001$ \\
\hline Ethnicity, $n$ (\%) & & & & $\chi^{2}(6)=21.97, P<0.001$ \\
\hline White & $346(68)$ & $169(83)$ & $41(77)$ & \\
\hline Asian & $93(18)^{*}$ & $17(8)^{* *}$ & $6(11)^{* *}$ & \\
\hline Black African-Caribbean & $44(9)^{*}$ & $7(4)^{\star *}$ & $2(4)^{* *}$ & \\
\hline Mixed ethnicity & $24(5)$ & $11(5)$ & $4(8)$ & \\
\hline DUP > 4 months, $n(\%)$ & $217(43)$ & $76(38)$ & $27(51)$ & $\chi^{2}(2)=3.64, P=0.16$ \\
\hline PANSS positive, mean (s.d.) & $15.63(5.85)^{\star}$ & $13.98(5.93)^{\star *}$ & $14.35(5.23)$ & $F(2,724)=6.04, P=0.002$ \\
\hline PANSS negative, mean (s.d.) & $16.16(6.73)^{\star}$ & $13.45(5.95)^{* *}$ & $12.00(4.59)^{* *}$ & $F(2,715)=18.92, P=0.001$ \\
\hline PANSS general, mean (s.d.) & $33.84(9.91)^{*}$ & $31.28(10.37)^{\star *}$ & $30.94(7.67)$ & $F(2,719)=5.73, P=0.003$ \\
\hline Calgary Depression Scale, mean (s.d.) & $6.47(5.44)$ & $5.91(5.16)$ & $7.13(5.31)$ & $F(2,733)=1.33, P=0.27$ \\
\hline \multicolumn{5}{|l|}{ Premorbid adjustment, mean (s.d.) } \\
\hline Childhood & $0.24(0.18)$ & $0.21(0.18)$ & $0.26(0.18)$ & $F(2,712)=2.05, P=0.13$ \\
\hline Early adolescence & $0.31(0.17)^{\star}$ & $0.25(0.17)^{\star \star *}$ & $0.25(0.16)$ & $F(2,688)=9.42, P<0.001$ \\
\hline Late adolescence & $0.35(0.19)^{*}$ & $0.24(0.17)^{\star *}$ & $0.23(0.15)^{\star \star *}$ & $F(2,583)=21.10, P=0.001$ \\
\hline
\end{tabular}




\begin{tabular}{|c|c|c|c|}
\hline & $B$ & s.e. & Odds ratio \\
\hline \multicolumn{4}{|l|}{ Moderate-Increasing v. Low Stable } \\
\hline Intercept & 0.39 & 0.67 & \\
\hline Age at onset of psychosis & 0.33 & 0.24 & 1.03 \\
\hline Positive symptoms & -0.06 & 0.03 & $0.95^{*}$ \\
\hline Negative symptoms & -0.03 & 0.02 & 0.97 \\
\hline General symptoms & -0.01 & 0.02 & 1.00 \\
\hline Depression & -0.01 & 0.03 & 0.99 \\
\hline Premorbid adjustment (up to 11 years) & 0.96 & 0.87 & 2.61 \\
\hline Premorbid adjustment (12-15 years) & -0.16 & 1.07 & 0.86 \\
\hline Premorbid adjustment (16-18 years) & -2.64 & 0.91 & $0.07 * *$ \\
\hline Gender (females $v$. males) & 0.48 & 0.24 & $1.61^{*}$ \\
\hline \multicolumn{4}{|l|}{ Ethnicity (base = White British) } \\
\hline Asian & -1.06 & 0.38 & $0.35^{\star \star}$ \\
\hline Black African-Caribbean & -1.74 & 0.58 & $0.18^{* *}$ \\
\hline Mixed ethnicity & 0.23 & 0.46 & 1.26 \\
\hline Duration untreated psychosis (short $v$. long) & 0.25 & 0.23 & 1.28 \\
\hline \multicolumn{4}{|l|}{ High-Decreasing v. Low Stable } \\
\hline Intercept & -2.63 & 1.10 & \\
\hline Age at onset of psychosis & 0.12 & 0.04 & $1.12^{* * *}$ \\
\hline Positive symptoms & -0.04 & 0.05 & 0.96 \\
\hline Negative symptoms & -0.10 & 0.05 & $0.91 *$ \\
\hline General symptoms & 0.02 & 0.04 & 1.02 \\
\hline Depression & 0.01 & 0.04 & 1.01 \\
\hline Premorbid adjustment (up to 11 years) & 2.83 & 1.44 & $17.00^{*}$ \\
\hline Premorbid adjustment (12-15 years) & -1.35 & 1.95 & 0.26 \\
\hline Premorbid adjustment (16-18 years) & -3.88 & 1.78 & $0.02 *$ \\
\hline Gender (males $v$. females) & 0.77 & 0.39 & $2.16^{*}$ \\
\hline \multicolumn{4}{|l|}{ Ethnicity (base = White British) } \\
\hline Asian & 0.05 & 0.56 & 1.05 \\
\hline Black African-Caribbean & -0.38 & 0.83 & 0.68 \\
\hline Mixed ethnicity & 0.37 & 0.84 & 1.45 \\
\hline Duration untreated psychosis (short $v$. long) & -0.44 & 0.39 & 0.64 \\
\hline
\end{tabular}

(7\%) also had a good outcome as their functioning remained in the non-clinical range for the duration of the study. However, this group experienced a large reduction in their time use over the 12-month period. This reduction is not necessarily problematic as this group were engaging in a very high level of activity at baseline, which then reduced to more normative levels. The exact reason for the high levels of activity at baseline requires further investigation but it could reflect over-activity resulting from mania or insomnia, which then stabilises. Alternatively, the trajectory could reflect a deteriorating profile which would be more problematic. Continuing to follow-up this group over a longer period of time would be useful in order to examine whether activity levels plateau or reduce further.

The rates of recovery outlined in this study are similar to those outlined in previous studies of FEP cohorts using alternative definitions and outcome measures. Wunderink et al ${ }^{22}$ report $26.4 \%$ of patients with FEP as functionally recovered after 2 years, with recovery defined as not experiencing any disability on any of the seven functional roles outlined in the Groningen Social Disabilities Schedule. Strakowski et al $^{23}$ define recovery as 8 weeks of functioning consistently at the premorbid level, and report a $35 \%$ recovery rate after 12 months following the first admission into hospital. However, premorbid functioning may not reflect a good outcome when compared with non-clinical groups. Indeed, a strength of the current study is the use of time use as a more explicit and defined measure of social functioning which can be directly compared with non-clinical norms. Nevertheless, the current study only focuses on functional outcomes and does not take into account symptomatic recovery.
The results of the current study suggest that functional recovery from FEP may be more difficult to achieve than symptomatic recovery, with previous research indicating over $50 \%$ of patients with FEP make a symptomatic recovery. ${ }^{22}$ In a recent meta-analysis, rates of recovery were only $14 \%$ when both clinical and social elements were included in the definition. ${ }^{24}$ More targeted intervention may be required to improve social recovery from early psychosis. Part of the role of EIP is to help individuals maintain their premorbid level of functioning ${ }^{25}$ and the findings suggest that this occurs for some individuals. However, individuals with poor premorbid functioning may require additional assistance to improve their functional outcome.

\section{Predictors of recovery}

The findings of this study support previous literature identifying gender ${ }^{26}$ ethnic minority status; ${ }^{27}$ younger age at illness onset; ${ }^{28}$ premorbid adjustment; ${ }^{29}$ and negative symptoms ${ }^{30}$ as predictors of poor social recovery following psychosis. It is possible that there is a common factor underlying all of these variables, influencing adaptation to psychosis and eventual social recovery. One question warranting further evaluation is the notion of social competence, referring to an individual's ability to impact favourably on their social world. ${ }^{31}$

Increased negative symptoms, poor adolescent premorbid adjustment, and a younger age at onset of psychosis may all reflect disruption in the development of the skills required to solve life problems and achieve instrumental and affiliative goals. Moreover, social networks have been found to be reduced in males ${ }^{32}$ and 
ethnic minorities, ${ }^{33}$ possibly indicating reduced social capital in these groups. This may influence the onset of psychosis but also an individual's resilience and the amount of interpersonal resources available in terms of coping with the consequences of a psychotic episode.

Interestingly, age at onset differentiated between Low Stable and High-Decreasing trajectories, with older participants more likely to be classified as high-decreasing than low stable, but not between low stable and moderate-increasing classes. This may reflect a higher baseline level of activity in individuals who are older when they develop psychosis. This is consistent with literature suggesting poorer premorbid functioning in individuals with a younger age at onset of psychosis. ${ }^{28}$ In addition, individuals with ethnic minority status were more likely to be classified in the Low Stable compared with the Moderate-Increasing trajectory. However, ethnicity did not differentiate between Low Stable and High-Decreasing trajectories. This could be taken to suggest that individuals with ethic minority status are less likely to experience an improvement in their functioning, consistent with literature suggesting a poorer outcome in this group. ${ }^{27}$ These hypotheses require further research in order for the role of age at onset and ethnicity on outcome to be better understood.

\section{Social recovery $v$. symptomatic recovery}

Baseline levels of positive psychotic symptoms assessed by the PANSS did not consistently predict functional outcome, although individuals in the Moderate-Increasing trajectory did have lower baseline scores on the PANSS Positive subscale. This supports literature suggesting that functional recovery can be independent from symptomatic fluctuations. ${ }^{34}$ This finding is also in line with patient literature outlining recovery as living a meaningful life even within the constraints of mental illness. ${ }^{35}$ Indeed, some individuals manage to return to a good level of functioning even if they still experience psychotic symptoms.

In addition, baseline levels of depression did not predict functional outcome. This finding contradicts previous studies highlighting increased levels of depression and low self-esteem in individuals with social recovery difficulties. ${ }^{36}$ However, depression levels were high in the current study, with all recovery groups scoring above the cut-off for clinical levels of depression at baseline. Such ceiling effects would make differences between the groups difficult to observe. It may be the case that it is persistent depression which predicts long-term social disability, rather than low mood observed in the early stages of psychosis. Further research is necessary to investigate this in more detail.

\section{Clinical implications}

The results of this study suggest that certain groups of people may be more at risk of long-term social disability than others, including males and individuals from ethnic minority groups. Moreover, high levels of baseline negative symptoms and poor premorbid adjustment, specifically in late adolescence, are also indicative of social disability on entry into EIP services and poor social recovery at 12 months, as is a younger age at onset of psychosis. Individuals displaying the characteristics found to be linked with social disability may require monitoring and targeted intervention in relation to their activity levels and functional recovery.

In line with previous research, it seems that at least for some people, functional disability may occur prior to the onset of FEP. Thus, further research focusing on the prodromal phase is needed. There may be an argument for early intervention at the first stages of social disability, rather than waiting for the onset of positive psychotic symptoms. ${ }^{37}$ Indeed, just as research on DUP suggests that untreated psychotic symptoms may be toxic in terms of symptomatic recovery, ${ }^{38}$ the finding that poor premorbid adjustment predicts later social disability suggests that untreated social functioning problems may be toxic for social recovery. Interventions prior to the onset of psychosis may involve detection and monitoring of individuals displaying early signs of social disability, as well as mental health difficulties. Indeed, this supports findings that the inclusion of reductions in role functioning improves the predictive validity of at-risk mental state criteria. ${ }^{39}$ Engagement will also be key with this client group who may find it difficult to access services due to a high level of social exclusion.

Although, social recovery is a central feature of EIP policy and recent clinical guidelines, ${ }^{25,40}$ the findings of this study suggest that it may be difficult to achieve. Targeted interventions are likely to be necessary in formulating social recovery difficulties and improving functional outcomes after the onset of psychosis. Existing interventions include supported employment, ${ }^{41}$ cognitive remediation, ${ }^{42}$ cognitive-behavioural therapy for negative symptoms, ${ }^{43}$ and social recovery-oriented cognitive-behavioural therapy, ${ }^{44}$ all of which have produced promising results. Peer support groups have also been found to be useful following the onset of psychosis in order to increase social networks. ${ }^{45}$

\section{Weaknesses and future research}

Although this study has highlighted several predictors of social recovery following FEP, it does not explain why or how these variables affect outcome. The study design was observational and therefore any relationship between patient characteristics is not necessarily causal. Future studies should focus on examining mediators of relationships between predictors and outcome. This will be important in identifying mechanisms of change and developing effective interventions. The current study focused on baseline predictors of outcome but did not consider factors which may contribute to recovery occurring after the onset of psychosis, such as engagement with services, the way in which individuals understood and coped with their psychotic episode, and treatment adherence. The latter is particularly relevant given the recent debate about whether lower doses of antipsychotic medication result in better outcomes. ${ }^{46}$ Future outcome studies should measure patterns of medication usage to investigate this further. In addition, depression was the only measure of mood in the current study, whereas anxiety is also prevalent in FEP and needs to be considered. Moreover, cognitive deficits have previously been linked with poor functional outcomes ${ }^{47}$ and the affect of this was not assessed in the current study.

Due to the retrospective nature of assessments of premorbid adjustment, a prospective study examining profiles of functioning using the TUS with individuals in the prodromal phases of illness would be useful in unpicking whether functional deficits highlighted in the current study were a consequence of the onset of psychosis or whether they existed premorbidly. Baseline assessments in the National EDEN study were conducted up to 3 months after being accepted into the EIP service. Thus, it was difficult to assess the impact of symptoms on functioning as the acute psychotic episode had usually been stabilised by the time of the baseline assessment.

Finally, a longer-term follow-up would enable the process of recovery from psychosis to be examined in more detail. Previous studies have examined recovery over a 2 - to 7 -year period. ${ }^{30}$ It may be the case that the first year of EIP is mostly about remission of symptoms and adjusting to the impact of the episode rather than functional recovery, which can take longer to achieve. ${ }^{48}$ The inclusion of additional time points would also enable non-linear 
models to be examined. This was not possible in the current study due to constraints of the study design meaning that only three time points were available for analysis. This is a limitation as change in social disability over time may be non-linear for some individuals. For example, functioning may fluctuate over time or plateau after an initial improvement.

This study has described the heterogeneous nature of social recovery in FEP using weekly hours in structured activity as an index of social functioning. The findings suggest a large proportion of individuals remain socially disabled after 12 months of EIP service provision. Predictors of social recovery were identified, suggesting that males and individuals from ethnic minority groups may be at risk of social disability following FEP. In addition, individuals with a young age at onset of psychosis, high baseline levels of negative symptoms, and poor adolescent premorbid adjustment may also be at risk. Individuals with one or more of these indicators may require close monitoring and targeted intervention in relation to improving their social outcome following FEP. Future research should focus on developing understanding of the way in which predictors affect outcome, thus identifying potential mechanisms of change to inform intervention development. Moreover, a longer period of follow-up is required to examine social recovery over the full duration of EIP and beyond. Examining changes in functioning during the premorbid and prodromal phases will also be important in understanding when social disability becomes a problem and identifying potential windows for intervention.

Jo Hodgekins, BSC, PhD, ClinPsyD, Norwich Medical School, University of East Anglia, Norwich, UK; Max Birchwood, PhD, DSc, University of Warwick, Gibbet Hill Road, Coventry, UK; Rose Christopher, BSC, Norwich Medical School, University of East Anglia, Norwich, UK; Max Marshall, MB BS, MD, University of Manchester, Manchester, UK; Sian Coker, BSC, DPhil, Norwich Medical School, University of East Anglia, Norwich, UK; Linda Everard, BSC, Birmingham and Solihull NHS Mental Health Foundation Trust, Birmingham, UK; Helen Lester, MB, BCH, MD (deceased) previously at the University of Birmingham, Edgbaston, Birmingham, UK; Peter Jones, PhD, FMedSci, University of Cambridge, Cambridge, UK; Tim Amos, MB BS, MRCPsych, University of Bristol, Bristol, UK; Swaran Singh, MBBS, MD, FRCPsych, DM, University of Warwick, Coventry, UK; Vimal Sharma, MD, FRCPsych, PhD, University of Chester, Cheshire and Wirral Partnership NHS Foundation Trust: Nick Freemantle, MA, PhD, University College London, London; David Fowler, MSC, CPsychol, University of Sussex, Brighton, UK

Correspondence: David Fowler, Psychology Department, University of Sussex Brighton BN1 9RH, UK. Email: d.fowler@sussex.ac.uk

First received 27 Jun 2014, final revision 29 Sep 2014, accepted 17 Nov 2014

\section{Funding}

M.B. and S.S. were part funded by the National Institute for Health Research (NIHR) throug the Collaborations for Leadership in Applied Health Research and Care for the West Midlands. M.B. and S.S. are part-funded by the NIHR Collaboration for Leadership in Applied Health Research and Care West Midlands. This article presents independent research funded by the NIHR under the Programme Grants for Applied Research programme (RP-PG-0109-10074). The views expressed are those of the authors and not necessarily those of the NHS, the NIHR or the Department of Health.

\section{Acknowledgement}

Helen Lester, our great friend and colleague, who was a co-Cl on National EDEN sadly died during the production of this paper. We thank all individuals who participated in this research.

\section{References}

1 Couture SM, Lecomte T, Leclerc C. Personality characteristics and attachment in first episode psychosis: impact on social functioning. J Nerv Ment Dis 2007; 195: 631-9.

2 Mangalore R, Knapp M. Cost of schizophrenia in England. J Ment Health Policy Econ 2007; 10: 23-41.
3 Cornblatt BA, Carrion RE, Addington J, Seidman L, Walker EF, Cannon TD, et al. Risk factors for psychosis: impaired social and role functioning. Schizophr Bull 2012; 38: 1247-57.

4 Harrison G, Croudace T, Mason P, Glazebrook C, Medley I. Predicting the long-term outcome of schizophrenia. Psychol Med 1996; 26: 697-705.

5 Fowler D, Hodgekins J, Howells L, Millward M, Ivins A, Taylor G, et al. Can targeted early intervention improve functional recovery in psychosis? A historical control evaluation of the effectiveness of different models of early intervention service provision in Norfolk 1998-2007. Early Interv Psychiatry 2009; 3: 282-8.

6 Silverstein SM, Bellack AS. A scientific agenda for the concept of recovery as it applies to schizophrenia. Clin Psychol Rev 2008; 28: 1108-24.

7 Menezes NM, Arenovich T, Zipursky RB. A systematic review of longitudinal outcome studies of first-episode psychosis. Psychol Med 2006; 36: 1349-62.

8 Birchwood $\mathrm{M}$, Lester $\mathrm{H}$, McCarthy $\mathrm{L}$, Jones $\mathrm{P}$, Fowler $\mathrm{D}$, Amos $\mathrm{T}$, et al. The UK national evaluation of the development and impact of Early Intervention services (The National Eden studies): study rationale, design, and baseline characteristics. Early Interv Psychiatry 2014; 8: 59-67.

9 Department of Health. Mental Health Policy Implementation Guide. Department of Health, 2001.

10 Short S. Review of the UK 2000 Time Use Survey. Office for National Statistics, 2006

11 Hodgekins J. Investigating the Psychological Typology of Social Recovery in Individuals with First Episode Psychosis. University of East Anglia, UK, Unpublished Doctoral Thesis, 2012.

12 Larsen TK, McGlashan TH, Johannessen JO, Vibe-Hansen L. First-episode schizophrenia: II. Premorbid patterns by gender. Schizophr Bull 1996; 22 257-69.

13 Lloyd-Evans B, Crosby M, Stockton S, Pilling S, Hobbs L, Hinton M, et al. Initiatives to shorten duration of untreated psychosis: systematic review. Br J Psychiatry 2011; 198: 256-63.

14 Cannon-Spoor HE, Potkin SG, Wyatt RJ. Measurement of premorbid adjustment in chronic schizophrenia. Schizophr Bull 1982; 8: 470-84.

15 Kay SR. Positive and Negative Syndromes in Schizophrenia: Assessment and Research. Brunner/Mazel, 1991.

16 Addington D, Addington J, Maticka-Tyndale E. Assessing depression in schizophrenia: the Calgary Depression Scale. Br J Psychiatry 1993; 163 (suppl): $39-44$

17 Nagin DS. Group-based Modelling of Development. Harvard University Press, 2005

18 Jung T, Wickrama KAS. An introduction to latent class growth analysis and growth mixture modelling. Soc Pers Psychol Compass 2008; 2: 302-17.

19 Muthen BO, Muthen LK. Integrating person-centered and variable-centered analyses: growth mixture modeling with latent trajectory classes. Alcohol Clin Exp Res 2000; 24: 882-91.

20 Muthen LK, Muthen BO. Mplus User's Guide. Muthen \& Muthen, 1998.

21 SPSS. SPSS Base 16.0 User's Guide. SPSS, 2007.

22 Wunderink L, Sytema S, Nienhuis FJ, Wiersma D. Clinical recovery in firstepisode psychosis. Schizophr Bull 2009; 35: 362-9.

23 Strakowski SM, Keck PE, McElroy SL, West SA, Sax KW, Hawkins JM, et al. Twelve-month outcome after a first hospitalisation for affective psychosis. Arch Gen Psychiatry 1998; 55: 49-55.

24 Jaaskelainen E, Juola P, Hirvonen N, McGrath JJ, Saha S, Isohanni M, et al. A systematic review and meta-analysis of recovery in schizophrenia. Schizophr Bull 2013; 39: 1296-306.

25 Birchwood M, Fowler D, Jackson C. Early Intervention in Psychosis: A Guide to Concepts, Evidence and Interactions. Wiley, 2002.

26 Cotton SM, Lambert M, Schimmelmann BG, Foley DL, Morley KI, McGorry P, et al. Gender differences in premorbid, entry, treatment, and outcome characteristics in a treated epidemiological sample of 661 patients with first episode psychosis. Schizophr Res 2009; 114: 17-24.

27 Morgan C, Kirkbride J, Hutchinson G, Craig T, Morgan K, Dazzan P, et al. Cumulative social disadvantage, ethnicity and first-episode psychosis: a case-control study. Psychol Med 2008; 38: 1701-15.

28 Ballageer T, Malla AK, Manchanda R, Takhar J, Haricharan R. Is adolescentonset first-episode psychosis different from adult onset? J Am Acad Child Adolesc Psychiatry 2005; 44: 782-9.

29 Addington J, Addington D. Patterns of premorbid functioning in first episode psychosis: relationship to 2-year outcome. Acta Psychiatr Scand 2005; 112: 40-6.

30 Milev P, Ho BC, Arndt S, Andreasen NC. Predictive values of neurocognition and negative symptoms on functional outcome in schizophrenia: a longitudinal first-episode study with 7-year follow-up. Am J Psychiatry 2005; 162: 495-506. 
31 Mueser KT, Bellack AS, Morrison RL, Wixted JT. Social competence in schizophrenia: premorbid adjustment, social skill, and domains of functioning. J Psychiatr Res 1990; 24: 51-63.

32 Preston NJ, Orr KG, Date R, Nolan L, Castle DJ. Gender differences in premorbid adjustment of patients with first episode psychosis. Schizophr Res 2002; 55: 285-90.

33 Bhugra D, Bhui K. African-Caribbeans and schizophrenia: contributing factors. Adv Psychiatr Treat 2001; 7: 283-91.

34 Agerbo E, Byrne M, Eaton WW, Mortensen PB. Marital and labor market status in the long run in schizophrenia. Arch Gen Psychiatry 2004; 61: 28-33.

35 Anthony WA. Recovery from mental illness: the guiding vision of the menta health service in the 1990s. Psychosoc Rehabil J 1993; 16: 11-23.

36 Gureje $\mathrm{O}$, Harvey $\mathrm{C}$, Herrman $\mathrm{H}$. Self-esteem in patients who have recovered from psychosis: profile and relationship to quality of life. Aust $N \mathrm{ZJ}$ Psychiatry 2004; 38: 334-8.

37 Fowler D, Hodgekins J, Arena K, Turner R, Lower R, Wheeler K, et al. Early detection and psychosocial intervention for young people who are at risk of developing long term socially disabling severe mental illness: Should we give equal priority to functional recovery and complex emotional dysfunction as to psychotic symptoms? Clin Neuropsychiatry 2010; 7: 63-71.

38 Marshall $\mathrm{M}$, Lewis $\mathrm{S}$, Lockwood $\mathrm{A}$, Drake $\mathrm{R}$, Jones $\mathrm{P}$, Croudace $\mathrm{T}$. Association between duration of untreated psychosis and outcome in cohorts of first-episode patients: a systematic review. Arch Gen Psychiatry 2005; 62: 975-83.

39 Valmaggia LR, Stahl D, Yung AR, Nelson B, Fusar-Poli P, McGorry PD, et al. Negative psychotic symptoms and impaired role functioning predict transition outcomes in the at-risk mental state: a latent class cluster analysis study. Psychol Med 2013; 43: 2311-25.
40 National Institute for Health and Care Excellence. Psychosis and Schizophrenia in Adults: Treatment and Management (Clinical Guideline 178). NICE, 2014.

41 Rinaldi M, Killackey E, Smith J, Shepherd G, Singh SP, Craig T. First episode psychosis and employment: a review. Int Rev Psychiatry 2010; 22: 148-62.

42 Wykes T, Reeder C, Landau S, Everitt B, Knapp M, Patel A, et al. Cognitive remediation therapy in schizophrenia: randomised controlled trial. Br J Psychiatry 2007; 190: 421-7.

43 Grant PM, Huh GA, Perivoliotis D, Stolar NM, Beck AT. Randomized trial to evaluate the efficacy of cognitive therapy for low-functioning patients with schizophrenia. Arch Gen Psychiatry 2012; 69: 121-7.

44 Fowler D, Hodgekins J, Painter M, Reilly T, Crane C, Macmillan I, et al. Cognitive behaviour therapy for improving social recovery in psychosis: a report from the ISREP MRC Trial Platform Study (Improving Social Recovery in Early Psychosis). Psychol Med 2009; 39: 1627-36.

45 Castelein S, Bruggeman R, van Busschbach JT, van der Gaag M, Stant AD, Knegtering $\mathrm{H}$, et al. The effectiveness of peer support groups in psychosis: a randomized controlled trial. Acta Psychiatr Scand 2008; 118: 64-72.

46 Wunderink L, Nieboer RM, Wiersma D, Sytema S, Nienhuis FJ. Recovery in remitted first-episode psychosis at 7 years of follow-up of an early dose reduction/discontinuation or maintenance treatment strategy: long-term follow-up of a 2-year randomized clinical trial. JAMA Psychiatry 2013; 70 : 913-20.

47 Leeson VC, Barnes TR, Hutton SB, Ron MA, Joyce EM. IQ as a predictor of functional outcome in schizophrenia: a longitudinal, four-year study of first-episode psychosis. Schizophr Res 2009: 107: 55-60.

48 Crumlish N, Whitty $P$, Clarke $M$, Browne S, Kamali M, Gervin M, et al. Beyond the critical period: Iongitudinal study of 8-year outcome in firstepisode non-affective psychosis. Br J Psychiatry 2009; 194: 18-24.

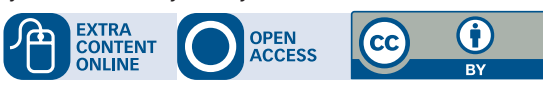

\section{0 wowa}

Verbatim

\section{David Brunskill}

'Word for word' examples should be revered for their subjective purity and revealing potential. Traditionally, forms of verbatim include the written: letters, poems, diaries and notes; and the spoken: audio, video and digital recordings. However, they also encompass accurately recorded and transcribed interviews and increasingly include modern forms of communication: email and social media outputs such as tweets, threads and wall postings. Even more abstract forms of personal expression and representation: painting, music, dance, sculpture etc., while usually regarded as visual arts in the first instance, may also share valuable potential as a gateway into elusive personal worlds of interest. 